\title{
Estudo Fitoquímico de Senna alata POR DuAs Metodologias ${ }^{1}$
}

\author{
Phytochemical Study of Senna alata Using Two Methodologies \\ RODRIGUES, I.M.C. ${ }^{2}$, SOUZA FILHO, A.P.S. ${ }^{3}$ e FERREIRA, F.A. ${ }^{4}$
}

\begin{abstract}
RESUMO - Senna alata, mais conhecida como mata-pasto na região Norte do Brasil, é uma planta utilizada pela medicina popular em várias partes do mundo e considerada espécie problemática em pastagens do Estado do Pará. No presente estudo, compararam-se duas metodologias distintas para a determinação das principais classes de constituintes potenciais aleloquímicos das diferentes frações (caules, flores, folhas, raizes, sementes e vagens) de S. alata. O material vegetal foi seco e sofreu extração exaustiva com solvente hidrometanólico, para obtenção dos extratos brutos; uma pequena parte dele foi solubilizada em metanol para obtenção das soluções utilizadas nos testes fitoquimicos. As metodologias utilizadas foram: testes por cromatografia em camada delgada (CCD) para determinação do perfil cromatográfico qualitativo; e ensaios para detecção preliminar dos diferentes constituintes químicos, com base na extração destes com solventes apropriados e na aplicação de testes de coloração. Os resultados em ambos os métodos demonstraram pouca semelhança, sendo o CCD o mais simples, barato, rápido e adequado para análise preliminar de compostos químicos derivados de plantas, embora seja um método qualitativo. Este método foi mais sensivel para a detecção de flavonoides, porém, para detecção de alcaloides, o reativo de Bouchardat foi mais sensivel do que o de Dragendorff, assim como o hidróxido de amônia $10 \%$ foi mais sensivel às antraquinonas do que o hidróxido de potássio. O estudo comprovou a alta diversidade de compostos químicos presentes em Senna alata, justificando sua ampla utilização na medicina popular e indicando ainda o potencial alelopático para a sua utilização.
\end{abstract}

Palavras-chave: aleloquímicos, cromatografia em camada delgada, fitoquímica, Leguminosae.

\begin{abstract}
Senna alata, known as mata-pasto in northern Brazil, is a plant used in popular medicine in several parts of the world, and considered harmful to pastures in the state of Pará. In this study, two different methodologies are compared to determine the main classes of potential allelochemicals of different fractions (stems, flowers, leaves, roots, seeds and pods) of S. alata. The plant material was dried and submitted to exhaustive extraction with hydromethanolic solvent to obtain the crude extracts, with a small part being solubilized in methanol to obtain the test solutions. The following methods were used: thin-layer chromatography (TLC), to determine the qualitative chromatographic profile, and preliminary detection tests of the distinct chemical constituents, based on their extraction with appropriate solvents and application of color testing. The results of both methods showed few similarities, with the TLC being the simplest, most inexpensive, fastest, and most appropriate for preliminary analysis of plant-derived chemical compounds, despite being a qualitative method. This method was more sensitive for flavonoid detection, although the Bouchardat reactive was more sensitive to alkaloid detection than the Dragendorff. Ammonium hydroxide $10 \%$ was more sensitive to anthraquinones than Potassium hydroxide. The study showed the high diversity of the chemical compounds present in Senna alata, justifying its extensive use in popularmedicine and even indicating its potential allelopathic use.
\end{abstract}

Keywords: Allelochemicals, Thin-layer chromatography, Phytochemistry, Leguminosae.

Recebido para publicação em 12.8.2008 e na forma revisada em 21.8.2009.

2 Mestranda do Programa de Pós-Graduação em Fitotecnia da Universidade Federal de Viçosa - DFT/UFV, 36570-000 Viçosa-MG, bolsista do CNPq, <izabellamcr@yahoo.com.br>; ${ }^{3}$ Pesquisador, Embrapa Amazônia Oriental, Trav. Dr., Enéas Pinheiro, S/N, 66095-100 Belém-PA; ${ }^{4}$ Professor do Departamento de Fitotecnia - DFT/UFV. 


\section{INTRODUÇÃO}

Na região Amazônica, as áreas de pastagens cultivadas são infestadas, historicamente, por comunidades de plantas daninhas, que têm por características a alta diversidade biológica e a agressividade, sendo esse um grande problema de ordem bioeconômica. Um bom exemplo de espécie considerada daninha é Senna alata, planta perene arbustiva, de crescimento rápido, pertencente à família Leguminosae, subfamília Caesalpinioideae, conhecida popularmente como mata-pasto na região Norte do Brasil. É frequente em áreas de pastagens, arredores de estradas e terrenos baldios, em quase todo o Brasil, principalmente em lugares úmidos, como a região de Belém (Lorenzi, 2000; PLANTAMED, 2007). Basicamente, essas comunidades promovem dois tipos de interferências nos cultivos que infestam: alelospolia e alelopatia. A primeira é observada nos ecossistemas devido à competição por fatores indispensáveis à sobrevivência das espécies, enquanto a alelopatia é promovida por agentes químicos produzidos e liberados para o ambiente pelas próprias plantas (Souza Filho \& Alves, 2002).

Teoricamente, todas as plantas são potencialmente capazes de sintetizar metabólitos secundários, como aleloquímicos. No entanto, essa característica é mais comum entre as plantas selvagens, que, ao longo do seu ciclo evolutivo, desenvolveram mecanismos de adaptação para competir com outras, assegurando sua sobrevivência quer pela formação de estandes puros, quer para ser defender de seus inimigos naturais (Souza Filho \& Alves, 2002). O potencial alelopático de extratos de Senna alata já foi investigado por Agbagwa et al. (2003), cujo estudo demonstrou seus efeitos sobre a inibição do crescimento da radícula e o decréscimo consistente no percentual absoluto e na taxa de germinação de Celosia argentea.

Senna alata possui também propriedades terapêuticas, devendo ser ministrada com cuidado, pois é suspeita de ser tóxica aos rins e, ainda, considerada abortiva (Lorenzi, 2000; PLANTAMED, 2007). Em Cuba, Camarões, no Brasil, na Índia e outros países, suas folhas, cascas, flores e raízes podem ser utilizadas na medicina popular, por suas propriedades anti-herpética, febrífuga, antianêmica, antiblenorrágica, antinefrítica, antídota, antimicótica, diurética, parasiticida, laxante, e contra doenças de pele (Barrese Pérez \& Hernández Jiménez, 2002; Awal et al., 2004; Barrese Pérez et al., 2005; Pieme et al., 2006; PLANTAMED, 2007).

Segundo Gupta \& Singh (1991), espécies do gênero Senna são ricas em flavonoides, antraquinonas e polissacarideos. Também já foram relatados para esse gênero esteroides, alcaloides piperidinicos, isoquinolinas, cromonas, lactonas, estilbenos e triterpenos (Alemayehu \& Abegaz, 1996; Alemayehu et al., 1998; Valencia et al., 2000).

A fitoquímica objetiva o esclarecimento e registro dos constituintes resultantes do metabolismo secundário dos vegetais, através do isolamento e elucidação de suas estruturas moleculares. O crescente desenvolvimento de novas técnicas analíticas, como a cromatografia, e o constante aperfeiçoamento dos instrumentos de análise espectrométrica constituem a sua principal força motora. A seleção de plantas para estudo, geralmente, se baseia na prospecção e em relatos da literatura sobre ações antioxidantes, anti-inflamatórias e inseticidas dos vegetais (Matos, 1997).

Desde muito tempo, a abordagem fitoquímica vem assumindo diferentes versões, ora mais simples ora mais complexa, mais especifica ou mais geral. O teste por cromatografia em camada delgada (CCD) tem por objetivo a caracterização preliminar dos extratos pela determinação do perfil cromatográfico qualitativo, onde se registra o número, a cor e a posição de manchas na placa (Rf) após a análise em sistemas diferentes de eluentes, associados aos reveladores específicos. A partir dessas avaliações, é possivel determinar as classes de compostos que estão presentes nos extratos analisados para permitir a posterior correlação com resultados obtidos nos testes de atividade biológica (Wagner \& Bladt, 1996; Gianvechio et al., 2006). Já o screening tradicional tem por objetivo a detecção e prospecção preliminar dos diferentes constituintes químicos de plantas, com base na extração destes com solventes apropriados e na aplicação de testes de coloração, ocorrendo algumas vezes a formação de precipitados (Moreira, 1979; Lock de Ugaz, 1988; Matos, 1997). 
Objetivou-se neste estudo a identificação, por testes fitoquímicos, das principais classes de compostos potenciais aleloquímicos, encontradas em diferentes partes da espécie Senna alata. Realizou-se ainda a comparação de duas metodologias distintas para este fim: a cromotografia em camada delgada e o screening padrão.

\section{MATERIAL E MÉTODOS}

\section{Coleta e processamento do material vegetal}

Senna alata foi plantada no Campo Experimental da Embrapa Amazônia Oriental, localizado na cidade de Belém, Pará. O plantio ocorreu em fevereiro de 2006. Uma amostra da espécie identificada por botânicos da mesma instituição foi depositada no herbário sob registro IAN 183561. Em dezembro de 2006, na fase reprodutiva, foram coletadas suas flores, vagens, sementes, raízes, folhas e caules. Em seguida, o material vegetal foi seco em estufa de circulação forçada de ar, à temperatura constante de $40{ }^{\circ} \mathrm{C}$, até atingir peso constante e então triturado em moinho tipo Willey até obtenção de um pó homogêneo.

\section{Preparo dos extratos brutos}

Para obtenção dos extratos brutos, realizou-se a extração exaustiva com mistura de solvente água/metanol (3:7) de cada parte da planta, por aproximadamente sete dias, com filtração e recolhimento periódicos do filtrado. Após esse período, o metanol foi eliminado a pressão reduzida, em rotavapor (BÜCHTEL 131) à temperatura de $45{ }^{\circ} \mathrm{C}$. Em seguida, a solução aquosa concentrada foi liofilizada.

Uma pequena parte do extrato bruto foi retirada e solubilizada em metanol, para obtenção das soluções utilizadas nos testes fitoquímicos (Wagner \& Bladt, 1996; Matos, 1997).

\section{Testes fitoquimicos}

O primeiro teste realizado foi o descrito por Wagner \& Bladt (1996) para a detecção de ácidos graxos, alcaloides, antraquinonas, cumarinas, flavonoides e terpenoides, via cromatografia em camada delgada.
Com o auxílio de tubos capilares de vidro, os extratos foram aplicados na origem de cinco cromatoplacas de sílica-gel (ALUMINUM BACKED TLC, SILICA GEL, HARD LAYER, F-254, SAI) de dimensões $5 \times 6 \mathrm{~cm}$, formando manchas semelhantes e regulares. Três placas foram eluídas com mistura de solvente polar ácido (acetato de etila: ácido fórmico: água 88:6:6). Uma placa foi eluída com mistura de solvente polar básico (tolueno: acetato de etila: dietilamina 70:20:10) e uma com eluente apolar (tolueno: acetato de etila 93:7). Após eluição, as placas foram secas e observadas sob luz de ultravioleta (UV), sendo registrados os resultados.

Visando evidenciar as principais classes de substâncias químicas presentes nos extratos retirados de diferentes estruturas da espécie $S$. alata, foram utilizados os seguintes reagentes (Wagner \& Bladt, 1996; Gianvechio et al., 2006; Maciel et al., 2006):

- DRG (Reativo de Dragendorff) e ácido sulfúrico $10 \%$, aplicados sobre a cromatoplaca eluída no sistema polar básico para a detecção de alcaloides (coloração amarronzada).

- NP/PEG (difenilboriloxietilamina/polietilenoglicol), aplicado sobre cromatoplaca eluída no sistema polar ácido para a detecção de cumarinas (azul-verde) e flavonoides (amarelo, laranja) sobre luz ultravioleta de comprimento de onda de $365 \mathrm{~nm}$.

- KOH (hidróxido de potássio), aplicado sobre cromatoplaca eluída no sistema polar ácido para a detecção de antraquinonas (vermelhoamarelo) e cumarinas (azul-verde) sobre luz UV $365 \mathrm{~nm}$.

- VAS (vanilina sulfúrica ácida), aplicada sobre cromatoplaca eluída no sistema polar ácido para a detecção de terpenoides (amarelomarrom) e ácidos graxos (azul).

- VAS (vanilina sulfúrica ácida), aplicada sobre cromatoplaca eluída no sistema apolar para a detecção de terpenoides (amarelo-marrom) e ácidos graxos (azul).

Os aspectos considerados foram cor e altura antes e após exame com luz UV e mudanças de coloração com o uso de reveladores.

- Cor: dependendo da tonalidade predominante, a cor da imagem em conjunto se define 
como vivamente colorida, pouco colorida ou muito pouco colorida.

- Mudanças de coloração: a imagem capilar foi exposta a reagentes sobre ela gotejados ou borrifados, e as mudanças de coloração foram observadas e registradas.

- Exame com luz ultravioleta: depois da corrida, a cromatoplaca é exposta à luz UV $365 \mathrm{~nm}$. Foram observadas e registradas as fluorescências das diferentes zonas da imagem e suas intensidades.

O segundo teste realizado foi o screening ou prospecção fitoquímica, descrito no Procedimento Operacional Padrão da Embrapa Amazônia Oriental, aplicado no setor de plantas medicinais do Laboratório de Agroindústria. Este procedimento utiliza técnicas descritas por Moreira (1979), Lock de Ugaz (1988) e Matos
(1997) (Tabela 1). Além dos constituintes testados nos procedimentos anteriores, foram também acrescentados os testes para taninos, catequinas, saponinas e depsídeos.

Todos os processos de extração, preparo de soluções e screening foram realizados no Laboratório de Agroindústria da Embrapa Amazônia Oriental, Belém, PA. Os testes em cromatoplacas de camada delgada foram realizados no Laboratório de Química da Universidade Federal do Pará.

\section{RESULTADOS E DISCUSSÃO}

O resultado da cromatografia em camada delgada pode ser observado na Tabela 2. Nesse caso, a ideia foi verificar a presença ou não de alcalóides, antraquinonas, cumarinas, flavonoides, terpenoides e ácidos graxos.

Tabela 1 - Testes de prospecção fitoquímica de classes químicas de plantas*

\begin{tabular}{|l|l|l|}
\hline Constituinte químico & \multicolumn{1}{|c|}{ Reagente } & \multicolumn{1}{c|}{ Reação positiva } \\
\hline Taninos & Solução aquosa de cloreto férrico a 1\% & Mudança de coloração ou formação de precipitado. \\
\hline Catequinas & $\begin{array}{l}\text { Solução aquosa de vanilina 1\% e ác. clorídrico } \\
\text { concentrado }\end{array}$ & Coloração vermelha intensa. \\
\hline Flavonoides & Ác. clorídrico concentrado e fita de Mg & Coloração rósea. \\
\hline $\begin{array}{l}\text { Esteroides e } \\
\text { Triterpenoides }\end{array}$ & $\begin{array}{l}\text { Clorofórmio, anidrido acético e ác. Sulfúrico } \\
\text { concentrado }\end{array}$ & $\begin{array}{l}\text { Sucessão de cores, de azul evanescente a v erde } \\
\text { persistente. }\end{array}$ \\
\hline Depsídios & Metanol e solução aquosa de cloreto férrico 1\% & Coloração verde, azul ou cinza. \\
\hline Cumarinas & Solução aquosa de NaOH 1 mol L em papel filtro. $^{-1}$ & Fluorescência azul sobre luz UV. \\
\hline Saponinas & $\begin{array}{l}\text { Solução hidroalcoólica 80GL: etanol comercial/água } \\
\text { destilada. }\end{array}$ & Camada de espuma estável por mais de 30 minutos. \\
\hline Alcaloides & Reativo de Bouchardat & Precipitado laranja avermelhado. \\
\hline Antraquinonas & Benzeno e solução aquosa de hidróxido de amônio 10\% & Coloração rósea, vermelha ou violeta na fase aquosa. \\
\hline
\end{tabular}

* Screening fitoquímico do Procedimento Operacional Padrão da Embrapa Amazônia Oriental.

Tabela 2 - Detecção de classes de substâncias químicas presentes nos extratos das diferentes partes de Senna alata pelo método de cromatografia em camada delgada (CCD)

\begin{tabular}{|l|c|c|c|c|c|c|}
\hline \multirow{2}{*}{ Classe química } & \multicolumn{6}{|c|}{ Extrato Bruto Hidrometanólico de Senna alata } \\
\cline { 2 - 7 } & Caules & Flores & Folhas & Raízes & Sementes & Vagens \\
\hline Ácidos graxos & + & + & + & + & + & + \\
\hline Alcaloides & - & - & - & - & - & - \\
\hline Antraquinonas & - & - & + & - & - & - \\
\hline Cumarinas & + & + & + & + & + & + \\
\hline Flavonoides & + & + & + & + & + & + \\
\hline Terpenoides & - & - & - & - & - & - \\
\hline
\end{tabular}

+ (positivo); - (negativo). 
Pelos resultados expostos, observa-se que cumarinas, flavonoides e ácidos graxos podem ser encontrados em todos os pontos da planta Senna alata. Antraquinonas foram detectadas somente nas folhas pelo teste com hidróxido de potássio. Ainda, as estruturas da planta que apresentaram maior diversidade de classes químicas, segundo o teste em CCD, foram as folhas, podendo essa diversidade refletir em um maior potencial de utilização em ensaios de alelopatia.

Já os ensaios para detecção e prospecção preliminar dos diferentes constituintes químicos de plantas, com base no screening fitoquímico do Procedimento Operacional Padrão da Embrapa Amazônia Oriental, forneceram os resultados expostos na Tabela 3.

Os resultados do screening mostram que alcaloides e antraquinonas foram encontrados em todas as estrututuras da planta, ao contrário do teste por $\mathrm{CCD}$, indicando que para detecção de alcaloides o reativo de Bouchardat foi mais sensivel do que o reativo de Dragendorff, assim como o hidróxido de amônia $10 \%$ foi mais sensivel às antraquinonas do que o hidróxido de potássio. Flavonoides não foram detectados utilizando-se ácido clorídrico concentrado e fita de magnésio, demonstrando que o NP/PEG (difenilboriloxietilamina/polietilenoglicol) é mais apropriado para detecção desta classe química. Da mesma forma, cumarinas só foram detectadas em raízes e sementes, sendo o hidróxido de potássio mais apropriado do que a solução aquosa de hidróxido de sódio. As folhas foram as únicas estruturas em que a presença de taninos foi confirmada. E contando com o resultado mascarado (indicando a suspeita da presença em baixas concentrações) para cumarinas e depsídios nas folhas, estas novamente, representaram a porção da planta que apresentou maior diversidade de classes químicas, como no teste em CCD. Saponinas também foram encontradas em praticamente todas as partes da planta.

Comparando-se as duas metodologias, poucos resultados foram corroborados, indicando que talvez a mistura de solvente água/metanol (3:7) utilizada na fase de extração não tenha sido a mais apropriada para algumas classes de compostos ou que a especificidade dos reagentes reveladores seja diferente para as mesmas concentrações. Esses resultados podem ainda estar relacionados à presença de intensa mistura de compostos e/ou à concentração muito baixa do constituinte a ser pesquisado nos tecidos da planta.

Os resultados obtidos na cromatografia de camada delgada demonstraram que esse é o método qualitativo mais adequado para uma análise preliminar de compostos químicos derivados de plantas, devido a economia, rapidez, simplicidade e maior segurança de manuseio do processo. Contudo, devem ser adicionados a esta metodologia mais testes para a abrangência de um maior número de classes, como no screening. Segundo Gianvechio et al. (2006) e Wagner \& Bladt (1996), das duas metodologias utilizadas, a cromatografia em camada delgada é considerada de fácil e rápida execução, custo

Tabela 3 - Detecção de classes de substâncias químicas presentes nos extratos das diferentes partes de Senna alata pelos métodos de prospecção fitoquímica de classes químicas*

\begin{tabular}{|l|c|c|c|c|c|c|}
\hline \multirow{2}{*}{ Classe química } & \multicolumn{6}{c|}{ Extrato Bruto Hidrometanólico de Senna alata } \\
\cline { 2 - 7 } & Caules & Flores & Folhas & Raízes & Sementes & Vagens \\
\hline Alcaloides & + & + & + & + & + & + \\
\hline Antraquinonas & + & + & + & + & + & + \\
\hline Catequinas & - & - & - & - & - & - \\
\hline Cumarinas & - & - & $\mathrm{M}$ & + & + & - \\
\hline Depsídios & + & - & $\mathrm{M}$ & - & - & - \\
\hline Flavonoides & - & - & - & - & - & - \\
\hline Saponinas & + & $\mathrm{M}$ & + & + & + & + \\
\hline Taninos & $\mathrm{M}$ & - & + & $\mathrm{M}$ & $\mathrm{M}$ & - \\
\hline Esteroides e triterpenóides & - & - & - & - & - & - \\
\hline
\end{tabular}

* Screening fitoquímico do Procedimento Operacional Padrão da Embrapa Amazônia Oriental; + (positivo); - (negativo); M (mascarado). 
acessivel, reprodutivel e consta em diversas monografias das Farmacopeias; é um método muito empregado uma vez que fornece dados para a identificação de matérias-primas vegetais, além de permitir inferências a respeito da pureza do material.

Neste trabalho, foram identificadas em todas as partes de Senna alata algumas classes químicas já consagradas pela atividade alelopática (Rice, 1984; Putnam \& Tang, 1986; Moreland \& Novitzky, 1987; Arruda et al., 2005; Cipollini, 2008). No entanto, classes como catequinas, esteroides e terpenoides, consideradas agentes alelopáticos potentes (Rice, 1984; Fischer et al., 1994; Lôbo et al., 2008; Macias, 2008), não foram encontradas. É reconhecido que as plantas produzem e estocam seus metabólitos em diferentes partes, sendo a principal fonte uma variável não fixa, que pode flutuar entre espécies ou dentro de uma mesma espécie e, ainda, sofrer variações de acordo com as condições ambientais (Souza Filho \& Alves, 2002).

Os resultados obtidos neste trabalho foram semelhantes aos encontrados por Barresse Pérez et al. (2005) no estudo fitoquímico da mesma espécie, onde também foram verificadas diferenças na detecção de constituintes químicos quando o solvente de extração foi modificado e até mesmo quando se utilizavam reagentes reveladores distintos. Entre os componentes até então identificados em Senna alata, por diferentes métodos fitoquímicos de prospecção, encontram-se taninos, triterpenos, esteroides, alcaloides, carboidratos redutores, flavonoides, saponinas, cumarinas, antocianidinas, emodina, antraquinona, chrysarabina, ribarina, ácido málico, tartárico, crisofânico e óleo essencial (Barrese Pérez \& Hernández Jiménez, 2002; Ordoñez et al., 2004; Barrese Pérez et al., 2005; PLANTAMED, 2007). Suas flores são ricas em flavonoides e vitamina C (PLANTAMED, 2007).

Neste estudo fitoquímico foi comprovada a alta diversidade de compostos químicos presentes em todas as estruturas de Senna alata, principalmente em suas folhas, justificando assim sua ampla utilização na medicina popular e indicando ainda o potencial para uso em outras áreas, como a alelopatia. Ainda, a cromatografia em camada delgada, devido à economia, rapidez, simplicidade e maior segurança do processo, é o método qualitativo mais indicado para a análise preliminar de compostos químicos derivados de plantas, como, por exemplo, potenciais classes de aleloquímicos.

\section{AGRADECIMENTOS}

Os autores agradecem ao $\mathrm{CNPq}$, ao Departamento de Fitotecnia da UFV, à Embrapa Amazônia Oriental e ao Departamento de Química da UFPA, Belém.

\section{LITERATURA CITADA}

AGBAGWA, I. O.; ONOFEGHARA, F. A.; MENSAH, S. I. Stimulation of growth and development of Celosia argentea L. by crude extracts of Senna alata (L.) Roxb. J. Appl. Sci.

Environ. Manag., v. 7, n. 1, p. 9-13, 2003.

ALEMAYEHU, G.; ABEGAZ, B. M. Bianthraquinones from the seeds of Senna multiglandulosa. Phytochemistry, v. 41, n. 3, p. 919-921, 1996.

ALEMAYEHU, G.; ABEGAZ, B.; KRAUS, W. A. 1-4, anthraquinone-dihydroantrhacenone dimer from Senna sophera. Phytochemistry, v. 48, n. 4, p. 699-702, 1998.

ARRUDA, M. S. P. et al. Potential allelochemicals isolated from Pueraria phaseoloides. Allelopathy J., v. 15, n. 2, p. 211-220, 2005.

AWAL, M. A. et al. Brine shrimp toxicity of leaf and seed extracts of Cassia alata Linn. and their antibacterial potency. J. Med. Sci., v. 4, n. 3, p. 188-193, 2004.

BARRESE PÉREZ, Y; HERNÁNDEZ JIMÉNEZ, M. E. Tamizaje fitoquímico de la droga y extracto fluido de la gacamaya francesa. R. Cub. Plant. Med., v. 7, n. 3, p. 1-4, 2002.

BARRESE PÉREZ, Y.; HERNÁNDEZ JIMÉNEZ, M. E.; PULPEIRO, O. G. Caracterización y estudio fitoquímico de Cassia alata L. R. Cub. Plant. Med., v. 10, n. 2, p. 1-5, 2005.

CIPOLLINI, D. et al. Phenolic metabolites in leaves of the invasive shrub, Lonicera maackii, and their potential phytotoxic and anti-herbivore effects. J. Chem. Ecol., v. 34, n. 2, p. 144-152, 2008.

FISCHER, N. H. et al. In search of allelopathy in the Florida scrubs: the role of terpenoids. J. Chem. Ecol., v. 20, n. 6, p. $1355-1380,1994$

GIANVECHIO, C. M. et al. Avaliação da qualidade de extratos vegetais. R. Eletrôn. Farm., v. 3, n. 2, p. 53-62, 2006. 
GUPTA, D.; SINGH, J. Flavonoid glycosides from Cassia alata. Phytochemistry, v. 30, n. 8, p. 2761-2763, 1991.

LÔBO, L. T. et al. Potencial alelopático de catequinas de Tachigali myrmecophyla (Legumnosae). Química Nova, v. 31, n. 3, p. 493-497, 2008

LOCK de UGAZ, O. Investigación fitoquímica: métodos en el estudio de productos naturales. Lima: Fondo Editorial Pontificia Universidad Católica del Perú, 1988. p. 1-7.

LORENZI, H. Plantas daninhas do Brasil: terrestres, aquáticas, parasitas, tóxicas e medicinais. 3.ed. Nova Odessa: Plantarum, 2000. $608 \mathrm{p}$.

MACÍAS, F. A. et al. Helikauranoside A, a new bioactive diterpene. J. Chem. Ecol., v. 34, n. 1, p. 6569, 2008.

MACIEL, R. L. et al. Características físico-químicas e químicas e estudo preliminar de estabilidade de tinturas preparadas com espécies de arnica Lychnophora em comparação com Arnica montana. R. Bras. Farmacogn., v. 16, n. 1, p. 99-104, 2006.

MATOS, F. J. A. Introdução a fitoquímica experimental 2.ed. Fortaleza: Edições UFC, 1997. 141 p.

MOREIRA, E. A. Marcha sistemática de análise em fitoquímica, Trib. Farmac., v. 47, n. 1, p. 1-19, 1979.

MORELAND, D. E.; NOVITZKY, W. P. Effects of phenolic acids, coumarins, and flavonoids on isolated chloroplasts and mitochondria. In: WALLER, G. R. (Ed.). Allelochemicals: role in agriculture and forestry. Washington: American Chemical Society, 1987. p. 247-261.
ORDOÑEZ, M. G;; GOVÍN, E. S.; BLANCO, M. A. G. Actividad antimicrobiana de Senna alata L. R. Cub. Plant. Med., v. 9, n. 1, p. 0-0, 2004.

PIEME, C. A. et al. Evaluation af acute and subacute toxicities of aqueous ethanolic extract of leaves of Senna alata (L.) Roxb (Caesalpiniaceae). Afric. J. Biotechnol., v. 5, n. 3, p. 283-289, 2006.

PLANTAS E ERVAS MEDICINAIS E FITOTERÁPICOS PLANTAMED. Disponível em: <http://

www.plantamed.com.br/plantaservas/especies/

Senna_alata.htm> Acesso em: 30 abr. 2007.

PUTNAM, A. R.; TANG, C. S. Allelopathy: state of the science. In: PUTNAM, A. R.; TANG, C. S. The science of allelopathy. Toronto: John Wiley \& Sons, 1986. p. 1-19.

RICE, E. L. Allelopathy. Orlando: Academic Press, 1984. $422 \mathrm{p}$

SOUZA FILHO, A. P. S.; ALVES, S. M. Alelopatia princípios básicos e aspectos gerais. Belém: Embrapa Amazônia Oriental, 2002. 260 p.

VALENCIA, E. et al. Estudio fitoquimico y atctividad antialimentaria de Senna stipulaceae. B. Soc. Chilena Química, v. 5, n. 2, p. 297-301, 2000.

WAGNER, H.; BLADT, S. Plant drug analysis - A thin layer cromatography atlas. 2.ed. Berlim: Springer, 1996. $384 \mathrm{p}$. 